\title{
Transatlantica
}

Revue d'études américaines. American Studies Journal

Line Breaks in America: the Odds and Ends of Poetry

\section{Retraduire The Waste Land : entretien avec Benoît Tadié}

\section{Chloé Thomas et Benoît Tadié}

\section{(2) OpenEdition}

1 Journals

\section{Édition électronique}

URL : https://journals.openedition.org/transatlantica/16879

DOI : 10.4000/transatlantica.16879

ISSN : 1765-2766

Éditeur

Association française d'Etudes Américaines (AFEA)

\section{Référence électronique}

Chloé Thomas et Benoît Tadié, "Retraduire The Waste Land : entretien avec Benoît Tadié »,

Transatlantica [En ligne], 1 | 2021, mis en ligne le 01 juillet 2021, consulté le 01 février 2023. URL:

http://journals.openedition.org/transatlantica/16879; DOI : https://doi.org/10.4000/transatlantica 16879

Ce document a été généré automatiquement le 1 février 2023.

Creative Commons - Attribution - Pas d'Utilisation Commerciale - Pas de Modification 4.0 International - CC BY-NC-ND 4.0

https://creativecommons.org/licenses/by-nc-nd/4.0/ 


\title{
Retraduire The Waste Land : entretien avec Benoît Tadié
}

\author{
Chloé Thomas et Benoît Tadié
}

\begin{abstract}
À propos de la parution de : T. S. Eliot, La Terre dévastée (The Waste Land), présentation et traduction de Benoît Tadié, Po\&sie, 2020/4 ( $\left.\mathrm{n}^{\circ} 174\right)$, https:// www.cairn.info/revue-poesie-2020-4-page-119.htm
\end{abstract}

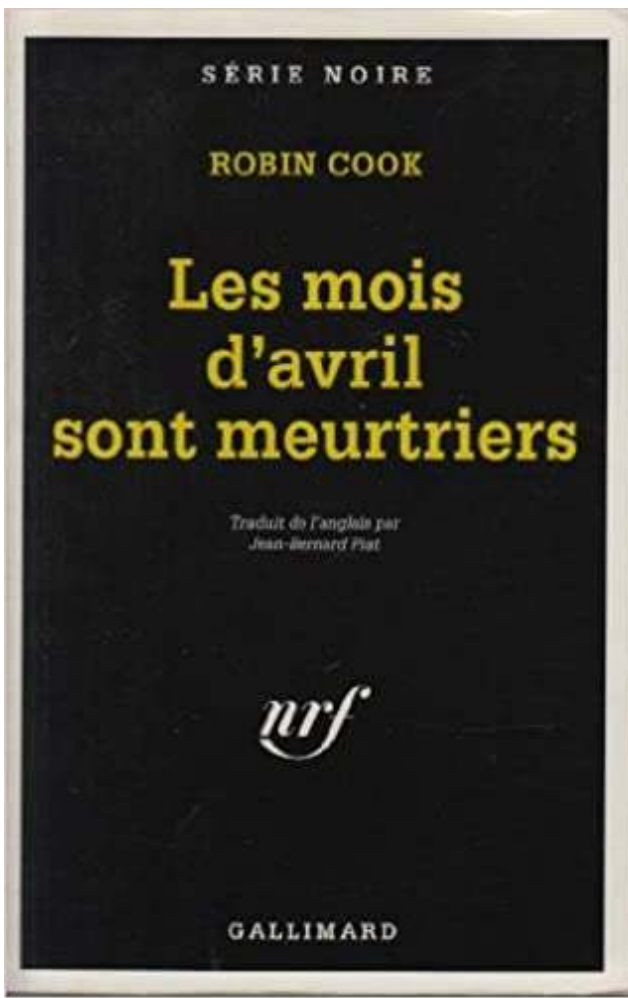

ChLó thomas : The Waste Land est un texte qui a déjà été traduit plusieurs fois et dont une édition française, celle de Pierre Leyris, est facilement disponible dans le commerce, en 
bilingue et en poche. Dans quel contexte as-tu fait cette traduction, et comment as-tu abordé le fait que ce soit une retraduction?

Benoît Tadié : Il y avait au départ un projet d'anthologie de la poésie américaine dirigée par Antoine Cazé et Christine Savinel pour la Pléiade, qui malheureusement n'a pas vu le jour. On m'avait demandé de faire quelques traductions pour ce projet, dont The Waste Land, mais aussi « Prufrock », " Gerontion »... J'avais donc traduit ces poèmes à l'époque, en 2007-2008, avant d'apprendre que malheureusement la Pléiade ne se ferait pas. Je les ai rangés dans mon ordinateur pour ne plus y toucher, jusqu'à rencontrer Martin Rueff, un des rédacteurs en chef de la revue Po\&sie, qui m'a proposé de publier des traductions de poésie américaine; j'ai donc ressorti ces traductions du placard. Évidemment, dix ans après, mon propre point de vue avait changé sur les textes; la question de la retraduction se posait avant tout pour moi par rapport à mon propre travail. Mais je me suis aussi rendu compte avec horreur que, quand on commence à vouloir transformer ce qu'on avait fait soi-même, tout se casse un peu la figure... Finalement, je n'ai pas beaucoup touché à ce que j'avais fait la première fois ; j'ai seulement affiné certains passages. De même, j'ai choisi d'ignorer le fait qu'il y avait d'autres traductions, dont une publiée dans Po\&sie aussi, certaines parues entre temps et celle de Leyris, qui est quand même assez ancienne et qui est celle qu'on trouve en exclusivité dans le commerce. Je me suis tenu à l'écart parce que je ne voulais ni faire la même chose, ni me forcer à faire autre chose si par hasard j'avais la même idée. Je me souvenais tout de même vaguement que Leyris avait un ton assez classique, qui correspond à l'époque où il a traduit ; je n'avais donc pas trop d'inquiétudes sur le fait que j'allais faire quelque chose d'assez différent, au moins sur le ton. De ce point de vue-là, je n'ai pas été gêné par le fait de retraduire, j'ai juste fait comme s'il n'y avait pas eu de traductions antérieures. Puis j'ai demandé conseil à quelques personnes, dont mon ancien directeur de thèse, Bernard Brugière, très fin connaisseur d'Eliot; j'ai relu toute la traduction un après-midi avec lui et il m'a fait beaucoup de suggestions que j'ai pu intégrer à ce texte.

CT : Tu as choisi d'intituler ta traduction La Terre dévastée, plutôt que La Terre gaste que l'on rencontre souvent dans la critique, ou La Terre vaine qui est le choix de Pierre Leyris. S'agissait-il pour toi de marquer un écart avec les traductions précédentes ?

BT : Pas seulement; je me suis d'ailleurs aperçu qu'une traduction précédente avait également choisi ce titre, ce que j'ignorais à l'époque. Surtout, je pensais que les mots comme «vaine», "inculte» ou "gaste», qu'on emploie habituellement, ne fonctionnaient pas pour différentes raisons. Par ailleurs, "dévasté » a le mérite d'avoir la même racine que «waste», comme "gaste», mais aujourd'hui plus personne ne sait ce que ça veut dire, "gaste», il faut un dictionnaire d'ancien français. J'ai donc voulu quelque chose qui reprenne le sens de « gaste » tout en étant un mot contemporain. « Dévasté » comme « gaste » viennent du latin «vastus » et du verbe « vastare » : « dévaster », " ravager ». J'avais d'ailleurs pensé à La Terre ravagée, mais c'était un peu trop guerrier. Dans notre contexte actuel de dévastation de la planète, l'adjectif prenait en outre un écho intéressant. Mais c'est surtout qu'il y a en anglais un verbe, " to waste », qui disparaît quand on choisit "vaine » ou " inculte », pas quand on choisit "dévastée ». On peut penser en ces termes de racines qui existent sous forme verbale ou adjectivale, ce qui est important pour la poétique moderniste, parce que chez des auteurs comme Pound en particulier, il y a tout une réflexion sur une sorte d'énergie verbale qui préexiste à la détermination des mots en tant que noms ou adjectifs, et qu'à la racine, justement, il y a des verbes. 
CT : Leyris, tu le soulignais, a fait une traduction classique ; peut-être parce qu'elle est assez tardive (1947) par rapport à ce texte qui avait entretemps acquis ce statut non seulement canonique, mais quasiment d'incarnation du canon. Peux-tu le resituer dans son contexte d'un point de vue formel ? Où en est-on en 1922 dans la poésie anglophone par rapport à ces questions de mètre, de vers libre?

BT : C'est difficile de donner une réponse générale car, en 1922, il y a beaucoup de choses différentes qui se passent, que l'on soit en Angleterre, en Amérique ou ailleurs. Mais par rapport au développement d'Eliot, de Pound et de ce groupe-là, ce qui précédait était le mouvement imagiste, d'ailleurs fondé aussi, chez Pound en particulier, sur l'idée de retraduction de passages antiques, où il s'agit moins de rendre accessible des passages antiques en anglais que de décentrer la forme poétique victorienne dont Pound essayait de se débarrasser. On le voit dans The Seafarer, un de ses premiers poèmes qui est un de ses meilleurs textes, traduit de l'anglo-saxon, dont la base est l'allitération : il essaye de faire une poésie purement allitérative, ça marche assez bien... Ensuite il a essayé de faire pareil avec le chinois, le latin, toujours d'une manière qui permette de créer des dissonances à l'intérieur du vers anglais. Chez Eliot on a un peu la même chose mais plutôt à partir du modèle symboliste français et aussi de l'idée du monologue dramatique, qui est chez lui et chez Pound très importante et qu'ils ont un peu empruntée à Browning. Il a aussi emprunté à Laforgue un ensemble de situations de communication plus varié et nouveau que les situations lyriques classiques de la poésie victorienne ou romantique. Il y avait une volonté de rompre avec le système victorien de Swinburne, de poètes qui avaient pu être d'ailleurs leurs premières amours poétiques. Eliot écrit quelque part de Swinburne que le pire qu'on puisse dire à son sujet c'est qu'à une époque on l'aimait et que désormais on ne l'aime plus : c'est que vraiment il est temps de passer à autre chose. Par rapport aux poèmes précédents d'Eliot (Prufrock, le premier recueil, en 1917, puis « Gerontion » en 1920), ce que The Waste Land apporte, c'est, pour la première fois, l'idée d'un poème long. Sauf certaines traductions et Hugh Selwyn Mauberley (1920) de Pound, les poèmes imagistes étaient fondés sur l'idée du fragment, ils étaient brefs et lapidaires, et redonnaient un poids à chaque mot plutôt que de faire des longs développements. C'est ce qu'on trouvait aussi chez T.E. Hulme, qui est mort très jeune à la guerre et n'a publié que quatre ou cinq poèmes, mais qui est une grande référence de Pound et Eliot. Avec The Waste Land, Eliot fait donc une avancée en passant à un poème long, fabriqué en réalité à partir d'un ensemble de poèmes courts, en produisant un effet de polyphonie extraordinaire pour l'époque, de dissonance, de différence, de rupture de style, de ton, de persona, qui font que pour beaucoup de gens en 1922, malgré la propagande active de Pound et d'un cercle autour de lui, c'était quelque chose de l'ordre de l'ovni qu'ils avaient du mal à reconnaître comme appartenant à la poésie ; c'est ce qu'on voit dans les premières critiques en tout cas.

CT : Dans ton introduction, tu cites la phrase de Pound, «to break the pentameter, that was the first heave». Est-ce que dans son travail d'édition du poème, il a poussé Eliot à aller encore plus contre l'aspect iambique?

BT : Oui, c'est très clair, il suffit de regarder dans l'édition facsimile combien il a coupé tout ce qui était de l'ordre de la régularité, notamment dans la quatrième partie, "Death by Water ", qui est assez classique dans sa forme. Ce n'est pas la seule chose contre laquelle il s'est acharné dans son travail d'édition : il a aussi coupé tout ce qui était à son sens trop grossier pour élever un peu le ton ; c'est ce qu'on a perdu 
dans l'édition définitive. Mais surtout, Pound a très certainement voulu accentuer les effets de rupture à l'intérieur du texte en supprimant les développements un peu trop longs, trop uniformes au sens étymologique d'une forme unique qui se développe sur le long cours. Il a été absolument impitoyable puisqu'il a réduit le travail d'Eliot à peu près de moitié et a créé un objet beaucoup plus moderne qu'il n'est dans les manuscrits.

CT : Y a-t-il des interventions proprement prosodiques de Pound, où il l'oblige à couper un vers?

BT : Oui, par exemple ici : "Unreal city, I have seen and see / Under the brown fog ", que Pound coupe : «Unreal city / Under the brown fog ». Au lieu d'un vers qui est à peu près régulier par rapport au suivant, on a juste deux pieds qui restent, avec un effet de réduction et de discontinuité beaucoup plus important. C'est amusant parce que Pound, à l'époque en tout cas, fait une poésie nettement plus classique que celle d'Eliot; mais comme éditeur il était beaucoup plus moderne, ils se sont bien complétés.

CT : Pourtant, Eliot a pu être vu par d'autres modernistes comme trop contraint par le rythme iambique (c'est ce que dit Hugh Kenner à propos de "Prufrock »), Williams en était très agacé...

BT : Williams était surtout agacé par l'idée d'une poésie internationale : il dit que ce poème a été une catastrophe, un désastre pour tout ce qu'il essayait de faire en vue d'une poésie américaine enracinée dans le localisme. Je pense que c'est plutôt en ces termes-là qu'il pose le problème. Je n'ai pas l'impression que dans les débats de l'époque la question du rythme iambique ait été très présente. Par ailleurs, on a une fausse idée rétrospective du classicisme du poème, ce qui est normal puisqu'il a été surexpliqué et patrimonialisé ; mais si on se remet dans les conditions de l'époque, le poème était quelque chose d'assez spécial et de non canonique. Cela est vrai pour Eliot aussi finalement, qui a toujours déprécié ce poème, qui ne voulait pas être connu pour ça ni par ça, et qui en parlait plutôt comme quelque chose dont il voulait se débarrasser pour passer à autre chose.

CT : II y a en tout cas dans tout le poème un jeu d'effacement du pentamètre iambique, qui est là de façon un peu spectrale: on rencontre parfois un vers isolé, ou une annonce de pentamètre qui va être cassé à la fin. Pour rendre cet effet, toi comme Leyris travaillez avec l'alexandrin, en considérant, peut-être parce qu'on n'a rien d'autre, que c'est ce qui correspond peu ou prou dans la prosodie française au pentamètre iambique. Est-ce par défaut ou y a-t-il un vrai recoupement des deux vers?

BT : Je pense qu'il y a une vraie isotopie, au sens où, à l'intérieur de chaque système et de chaque culture, ils représentent à peu près la même chose, ce qui ne veut pas dire que formellement ils fonctionnent de la même façon, bien sûr. Quand on a un pentamètre iambique, on pense tout de suite à Shakespeare et, pareillement, l'alexandrin convoque immédiatement Racine, Hugo: les deux vers contiennent l'évocation ou la connotation d'un patrimoine central de la poésie nationale.

CT : Mais si l'on parle de tradition, le pentamètre peut évoquer aussi bien Shakespeare que Wordsworth, et l'alexandrin, Racine que Hugo. Concrètement, en jouant avec le pentamètre iambique, avec quelle tradition particulière Eliot joue-t-il ?

BT : Quand on lit ses textes critiques, on voit qu'Eliot s'intéresse à une tradition qu'on peut définir sur un plan politique et religieux: il ne s'intéresse pas à la grande tradition de Milton et des romantiques, pour des raisons idéologiques comme on sait, mais à des traditions plus catholiques. Mais quand il cite Donne ou la poésie 
métaphysique, il va toujours chercher les poètes les plus excentriques par rapport à la tradition du pentamètre. Et même quand il s'intéresse aux dramaturges élisabéthains, il parle bien sûr avec révérence de Shakespeare, mais il est plus intéressé par des auteurs comme Webster, dont il montre bien les libertés qu'ils prennent avec le vers, dans les moments d'émotion intense où ils s'écartent du pattern. C'est ça qui l'intéresse : la dialectique entre la forme et l'absence de forme. Pour lui, soit on part de l'informe et on essaie de rapprocher ce chaos d'une forme simple et reconnaissable, soit on part d'une forme classique avec laquelle on prend des libertés dans les moments stratégiques. Mais il y a toujours un jeu avec la forme, jamais l'idée d'un vers qui serait purement libre; d'ailleurs, pour Eliot, il n'y a pas de liberté en littérature et pas de liberté dans l'art. Il n'y a pas de liberté dans le mètre, il n'y a que la maîtrise du mètre.

CT : En le traduisant, avais-tu en tête un type particulier d'alexandrin ? Celui de Racine?

BT : Plutôt Baudelaire, pour moi, parce qu'il cite Baudelaire et parce que je pense que l'univers de The Waste Land est assez proche de celui des "Tableaux parisiens », c'està-dire quelque chose d'à la fois réaliste et fantasmagorique qui correspond aussi à l'univers de la ville moderne, et puis parce qu'il n'y avait pas vraiment eu de poésie de la ville moderne en anglais avant Eliot. Il y a quelques précurseurs ou poèmes ici ou là mais pas grand-chose avant lui.

CT : Leyris, dans sa traduction, fait parfois des alexandrins abusifs, au sens où il retombe dans ce vers au-delà des cas où il cherche à retranscrire un pentamètre iambique. Du fait sans doute d'avoir traduit le texte à un moment où il était déjà canonisé, après le Nobel d'Eliot, il le sur-classicise un petit peu, et surtout il fait des alexandrins qui me semblent très raciniens. À plusieurs endroits je me suis demandé si on n'avait pas au contraire quelque chose de proche de Hugo, puisque Hugo c'est l'art d'enjamber, de casser, ce qui fait écho, sous certains aspects, au pentamètre iambique tel qu'il a pu être utilisé dans le Prélude, non plus une sortie du discours et une élévation par la poésie, mais la restitution du discours oral dans sa forme la plus pure.

BT : Eliot fait quelque chose d'assez différent; ce qui me frappe chez lui, c'est qu'il crée des effets de dissonance, par exemple entre la forme du pentamètre, qui est une forme noble et traditionnelle, et son contenu, ou bien le registre de langage, en cherchant à faire s'entrechoquer les deux. Il le fait beaucoup plus que Hugo : même si on cite souvent Hernani comme exemple de démocratisation de l'alexandrin, on est assez loin de ce genre d'effet. C'est ce qu'on trouve à la fin de la deuxième partie avec les dames qui discutent au pub et parlent d'avortement, un contenu jugé antipoétique à l'époque ; de l'autre côté on a des pentamètres, et le fait de coller l'un sur l'autre crée un effet de dissonance assez puissant. C'est pour ça qu'en français il faut utiliser plus le passé composé quand on traduit, parce que c'est un temps antipoétique qui contredit l'idée de l'alexandrin. L'alexandrin, c'est tout un protocole, pas juste douze syllabes.

CT: Dans ta traduction, j'ai repéré un quasi alexandrin : « si rudement outragé, mais là, le rossignol » qui demande, pour être lu comme un alexandrin, à faire une élision du $-\mathrm{e}$, "rud'ment»; ça oblige à une oralisation, d'une façon qui ne fait pas tant romantique que troubadour, chanson...

BT : Je me suis amusé à faire des vers de treize syllabes, ou onze...

CT : Pour toi c'est donc un vers de 13 syllabes ; il faut le lire « ru-de-ment »?

BT : Oui. Eliot ne pense pas à casser le système de la prosodie, puisqu'il ne pense pas qu'il y ait de la poésie en dehors de la prosodie, même si ça doit être une prosodie 
beaucoup plus désarticulée; de même, il faut raisonner en termes d'une prosodie classique française qui fonctionne par syllabes et rester dedans. C'est pour ça que ce serait une erreur de faire des élisions, même si ça peut se retrouver chez Laforgue et Corbière parfois.

CT : Eliot connaissait très bien ce corpus français, à partir de Mallarmé, mais sans vraiment prendre en compte le fait qu'ils écrivaient en opposition à l'incarnation de l'alexandrin qu'était Hugo.

BT : Je crois qu'il n'avait pas une connaissance assez fine du français; et je crois qu'il ne comprenait pas vraiment Mallarmé, il ne s'y intéressait pas, pas plus que Pound. Laforgue l'intéressait beaucoup plus, mais toujours par rapport à la possibilité d'utilisation d'un système laforguien en anglais.

CT: Les quelques poèmes d'Eliot en français sont d'ailleurs en alexandrins assez maladroits...

BT : En réalité ce sont des pentamètres : quand on les lit, ils se scandent comme en anglais. Il y a un modèle linguistique qui surdétermine ses vers. Mais, dans The Waste Land, il n'y a pas que l'alexandrin, il y a des sonnets entiers qui sont cachés dans le texte. Dans la troisième partie, il y en a un qui commence du début, «The river's tent is broken ", jusqu'à " spread from ear to ear » : c'est bien un sonnet qui se découpe en trois quatrains et un couplet. Et un peu plus loin, en voilà un qui constitue un bon exemple d'opposition entre la forme et le fond, à la fin de la scène entre la dactylo et l'agent immobilier: depuis "The time is now propitious» (v. 235) jusqu'à "finding the stairs unlit». On a un parfait sonnet avec des rimes $A B A B$, et puis un couplet final qui justement est dissonant parce que la dernière rime qu'on attend est imparfaite, « kiss» et "unlit». Ça crée une dissonance assez forte avec une forme qui a été parfaitement respectée jusque-là, qui est celle du sonnet shakespearien. Ce que je trouve fascinant, et il le dit dans son essai assez précoce "Réflexions sur le vers libre ", qui est son meilleur texte sur la poésie à mon sens, c'est la manière dont les formes se cachent derrière la tenture, un peu comme dans Hamlet, comme quelque chose aux aguets ; il faut que le lecteur puisse les deviner sans les voir complètement, que ça affleure sans être vraiment visible; et ça affleure souvent à des moments où ça crée, quand on s'en aperçoit, une dissonance très forte. Le sonnet est traditionnellement associé à l'amour courtois, à l'élévation spirituelle, et là on a exactement le contraire dans le contenu.

CT : Ce rapport à la métrique renforce aussi l'antagonisme entre Pound et Eliot, en tout cas en termes de posture. Eliot est finalement cohérent avec ce qu'il dit dans «Tradition and the Individual Talent », alors que Pound est dans une logique ouvertement conflictuelle avec les formes précédentes, et pourtant ce n'est sans doute pas celui qui fait le plus d'expérimentations formelles.

BT : La raison pour laquelle Eliot va plus loin c'est, je pense, qu'il est davantage capable de s'oublier lui-même dans sa poésie, «impersonnelle » comme il le dit luimême. À propos de Pound en revanche, McLuhan montre dans l'essai "Pound's Critical Prose ${ }^{1} »$ que sa poésie a fini par avaler sa prose critique. Il n'y a pas de différence entre les deux. Chez Eliot en revanche, on pourrait très bien dire que les essais n'ont pas été écrits par le poète et inversement, même si on peut les mettre en rapport. On sent qu'il est capable d'oublier ce qu'il est dans sa poésie, et c'est pour ça qu'il a eu plus de succès comme poète, et Pound comme critique. En tout cas Pound a eu une influence beaucoup plus grande comme organisateur du mouvement poétique, comme éditeur. 
CT : Si l'on revient à la syntaxe, ta traduction est assez « vers par vers » par rapport à celle de Leyris, et donc proche de la syntaxe anglaise. Est-ce un choix conscient?

BT : Je voulais aller vers par vers et même mot par mot, pour être le plus proche possible du texte, avec l'idée qu'il avait été beaucoup normalisé, domestiqué en français et qu'il fallait faire ressortir son côté un peu incongru, et plus polysémique, polyphonique, discontinu, plus abrupt aussi, que ce qu'on a en français. Quand on voit la première réaction de certains critiques, dont celui que je cite en introduction, ce F.L. Lucas ${ }^{2}$ qui est resté célèbre pour n'avoir pas compris que The Waste Land était un seul poème, il fallait qu'on puisse se dire aussi face au texte français que c'est une collection de fragments, de parodies, d'imitations. Or quelqu'un qui lirait la traduction de Leyris ne pourrait pas imaginer que ce n'est pas un seul poème. Il fallait le traduire dans cet esprit-là, d'où aussi le côté détachable, discontinu, le changement du passé simple au passé composé ; j'ai essayé de heurter beaucoup plus l'oreille du lecteur.

CT : Leyris a tendance à lisser, notamment les enjambements, ce que tu ne fais pas. Mais dans ta traduction, il y en a un que j'ai trouvé intéressant, assez hardi : "They expect / nothing»; «Qui attendent / rien ». J'imagine que tu as voulu garder l'effet de surprise que crée l'alinéa, mais au prix d'un écart grammatical.

BT : C'est un peu comme chez Beckett: "rien », c'est aussi un mot, donc aussi quelque chose. Je pense que le rien est assez plein : "Do / you know nothing », par exemple, dans la deuxième partie, ça peut aussi vouloir dire " connaître le néant »; il y a une ambiguïé, et le sens fort est peut-être plus intéressant que « ne sais-tu / Rien ? Ne vois-tu rien? » : j’aurais pu enlever la négation, « Sais-tu / Rien ? Vois-tu rien ?»...

CT : Ce serait une traduction quasi-beckettienne, justement..

BT : Je me suis dit que ça pouvait marcher, d'autant plus qu'il y a un poème de William Carlos Williams très intéressant qui joue justement sur cette ambiguïté du " rien » comme «no-thing ", comme présence d'un vide, un poème qui s'appelle «To have done nothing ", qui n'est pas la même chose que "Not to have done anything" quand on y réfléchit : " avoir fait / rien ». Quand on lit ce poème de Williams qui date des années vingt aussi, un peu après celui d'Eliot, on voit que c'est une façon de penser qui n'était pas impossible à l'époque.

CT : Tu parlais de casser la fausse impression d'unité que pouvait donner la traduction de Leyris. T'es-tu efforcé, en préambule à l'acte de traduire, de départager les voix ?

BT : Je l'ai toujours fait car j'ai beaucoup enseigné ce texte. Les changements sont assez nets. Je n'ai jamais avalé l'idée que toutes les voix se rejoignent dans Tiresias, ce qu'Eliot dit dans ses notes; je pense que ça, c'est une espèce de reconstruction $a$ posteriori pour recréer un effet d'unité qu'on n'a pas intérêt à recréer finalement, car ce n'est pas l'intérêt du poème à mon avis.

\section{CT : Comment les as-tu séparées dans ta traduction?}

BT : Par le registre, bien sûr; par exemple si on prend le début, le premier changement advient avec «L'été nous a surpris ", d'où le passage au passé composé.

CT : Il y a mélange des voix, et donc aussi mélange des langues. Leyris laisse Shakespeare en anglais dans le texte, peut-être avec la supposition qu'un lectorat français cultivé va 
reconnaître les vers de Shakespeare, ou avec l'idée de garder une part d'étrangeté. Toi, tu traduis plutôt Shakespeare.

BT : La question des vers étrangers - étrangers au texte, pas forcément étrangers à l'anglais - est très intéressante. C'est difficile de savoir, quand un vers est cité, si le vers est mentionné ou utilisé, et c'est ça qui pousse soit à traduire l'emprunt, si on estime qu'il est intégré au texte, soit à ne pas le traduire si c'est plutôt un fragment, une citation. Quand c'est la même langue, à mon sens, il faut tout traduire ; je traduis presque tous les emprunts de l'anglais, sauf "That Shakespeaharian rag » que j'ai mis en italiques, parce que c'est une citation d'une chanson : si on avait traduit « ce rag shakespeahirien ", on n'aurait pas vu la chanson. "London bridge is falling down » est laissé tel aussi : c'est un vers de comptine. Il faut aussi distinguer les vers qui sont cités intacts et ceux qui sont un peu tordus. Quand ils sont tordus, il faut les traduire parce que s'il y a une torsion il y a une vraie réappropriation du vers. Si c'est là en tant que tel, comme pour «London Bridge », il y a un tel rythme musical que ça aurait été dommage de l'enlever. "Sweet Thames, run softly » est cité puis varié ensuite, donc je l'ai traduit. C'était assez pragmatique comme approche.

CT : Ne pas traduire la chanson, «Shakespeaharian rag » ou «London bridge », fait aussi de l'anglais une langue pop, alors que Leyris la prend comme une langue de culture, la langue de Shakespeare au sens propre. Par ailleurs, quand Eliot cite un vers en français dans le texte, tu le mets en italiques; Leyris le laisse en romaines, comme si le lecteur français devait reconnaître la citation sans qu'elle soit signalée.

BT : Là il faut vraiment marquer la différence linguistique ; or on ne peut pas mettre une note qui dirait «en français dans le texte». D’ailleurs les vers français deviennent vraiment des vers anglais : l'emprunt à Baudelaire devient « You, hypocrite lecteur, mon semblable, mon frère". L'ajout de "you» recrée un pentamètre qui autrement ne fonctionnerait pas : on ne pourrait pas le scander. Avec «you » devant ça nous fait un trochée ou un spondée, et on peut alors avoir cinq pieds : ce « you » est là pour interpeller le lecteur mais aussi pour des raisons, simplement, de scansion.

CT : Cette question des langues touche à ce que serait une traduction moderniste de ce texte.

BT: On sait ce que sont les traductions modernistes faites par les poètes modernistes : elles ont une très grande liberté par rapport à l'original. C'est ce qu'on ne peut pas se permettre quand on est soi-même traducteur d'un texte canonique... J'ai toujours trouvé que le premier vers, "April is the cruellest month, ... », sonne mal en français : "Avril est le mois le plus cruel», ça ne coule pas; mais on est tenu par une fidélité au sens. J'aurais aimé changer le vers : il y a un roman noir anglais que j'aime beaucoup, un bouquin de Robin Cook dont le titre français est Les mois d'avril sont meurtriers. "Les mois d'avril sont meurtriers ", c'est un super premier vers ; j'ai failli le mettre, mais je me suis dit que si je commençais il n'y avait plus de limite, ce serait faire comme Pound dans Hommage to Sextus Propertius où il avait transformé ce qui l'amusait, y compris en rajoutant des contre-sens volontaires du moment que ça permettait des formulations amusantes.

CT : Ça n'a jamais été fait, une traduction moderniste de The Waste Land?

BT : Non, ça n'aurait pu être fait que par un poète.

CT : Et les poètes français se sont moins intéressés à Eliot...

BT : Ils avaient Apollinaire. Sans se ressembler, Eliot et lui jouent un rôle comparable, un peu comme le pentamètre et l'alexandrin : ils ont un rôle de charnière entre un 
système classique et un système moderne. Comme Eliot, Apollinaire montre une maîtrise éblouissante du vers classique et de la prosodie classique ; on voit que seul quelqu'un qui connaît aussi bien le système classique a pu le casser aussi bien.

CT: Pourtant, contrairement à d'autres poètes modernistes, Eliot a bénéficié d'une traduction, celle de Leyris, qui est assez juste.

BT : Il y a une erreur sémantique, la seule peut-être, dans la traduction de Leyris. C'est dans la troisième partie: "Sweeney to Mrs Porter in the spring» (v. 198). Il a traduit « in the spring " par « au printemps », alors que c'est « dans la source». Eliot l'indique dans la note : c'est une parodie de la scène avec Diane et Actéon dans The Parliament of Bees de John Day. Comme on a un poème qui ne parle que du printemps, on a tendance à plaquer des interprétations, mais l'avantage de faire du mot à mot c'est de considérer que « spring " peut aussi être la source, et la référence à Diane et Actéon le confirme.

CT: À propos des notes, celles d'Eliot, dans l'édition bilingue de Leyris, ne sont pas en bilingue: elles sont considérées comme des notes d'édition, et d'ailleurs mélangées avec celles de John Hayward, écrites pour l'édition française. De ton côté, tu les as abordées comme faisant partie du poème?

BT : Oui, les notes font partie du poème, ne serait-ce que parce qu'elles sont assez arbitraires, n'éclairent pas forcément grand-chose, envoient parfois même sur de fausses pistes : elles sont une voix de plus dans le poème, qu'il faut traduire comme telle.

CT : Tu n'as pas voulu rajouter tes propres notes?

BT : Non, d'ailleurs cela n'aurait pas été possible dans le cadre d'une publication en revue, quand on voit les proportions que prennent aujourd'hui les éditions annotées.

CT : II n'existe justement pas d'édition scientifique d'Eliot en français. Mais il est accessible dans cette traduction de Leyris qui est donc, de fait, l'édition de référence.

BT : Sa traduction est belle, mais elle émousse complètement le texte : on ne voit plus que c'est un poète moderne. On voit une sorte de Valéry, avec ici et là quelques libertés, mais on n'arrive pas du tout à comprendre en quoi c'est moderniste, si on redonne un peu de force à ce mot. On manque surtout le fait que la poésie plus tardive d'Eliot l'est beaucoup moins : ça s'aplatit à partir des Quatre quatuors. Dans l'édition de Leyris, on ne voit pas qu'il y a eu ce moment assez révolutionnaire suivi par un moment d'institutionnalisation. C'est ce qu'il faudrait faire ressortir si on retraduisait Eliot aujourd'hui : qu'il y a une première phase qui s'arrête à peu près à Sweeney Agonistes, le dernier poème moderniste; ensuite il y a tout le reste, admirablement écrit parfois, mais pas du tout dans la même logique subversive.

CT: Est-ce que ça a joué dans la réception française du corpus moderniste, cette incarnation du modernisme dans ce poème-là d'une part, et le fait qu'on ait accès à ce texte dans cette traduction-là d'autre part?

$\mathrm{BT}$ : Le poème a été traduit tard et, finalement, il y a eu très peu de traductions contemporaines d'Eliot, même s'il y a eu celle de «Prufrock» dans Le Navire d'argent en 1925, par Sylvia Beach et Adrienne Monnier. Mais comme pour Pound, il y a eu un effet de retard énorme, et c'est seulement à partir des années cinquante ou soixante qu'ils commencent à être considérés en France, à un moment où ils ne peuvent plus apporter grand-chose. Pour Pound c'est d'autant plus frappant qu'il vivait à Paris au début des années vingt, qu'il y était correspondant de revues, qu'il disait fréquenter les milieux artistiques parisiens dont il rendait compte de façon un peu particulière : 
en réalité il était totalement déconnecté, pas traduit, il avait peut-être quelques fréquentations de salon mais il n'a pas percé dans la culture française avant les années soixante. C'est ce qu'a bien montré la thèse récente de Jean-Christophe Contini sur la réception de Pound en France et en Suisse : elle commence vraiment à partir d'un double Cahier de l'Herne de 1965-1966 d'une part, et des traductions par Denis Roche, membre du groupe Tel Quel, d'autre part, à peu près au même moment. Il y a donc une réception de gauche et une réception de droite, quasi concomitantes. Dans l'Herne, le double cahier sur Pound venait juste après celui sur Céline, donc ça a tout de suite assimilé Pound au débat sur Céline, et c'est à travers ça qu'on l'a perçu en France. De l'autre côté, il y avait la lecture impulsée par Denis Roche, Sollers, etc., qui ont toujours défendu Pound comme une espèce de martyr. Au moins Denis Roche a fait l'effort de le traduire. Dominique de Roux, éditeur de l'Herne notamment, l'a fait venir en France : on trouve des documents sur YouTube montrant Pound à Paris accompagné par Dominique de Roux ${ }^{3}$, avec des jeunes qui s'agitent autour de lui et lui complètement enfermé dans ses pensées, ne parlant quasiment plus...

CT: On retrouve l'aspect totémique de Pound, forcément attaché à sa personne; alors qu'avec Eliot c'est le texte qui rentre le canon... C'est bientôt, d'ailleurs, l'année du centenaire. Est-ce que c'est un texte qui peut encore être lu comme en rupture, malgré son hyper-canonisation?

BT : Absolument. Il faut en avoir une lecture historique, comme quand on regarde un Chaplin ou qu'on lit Hernani. Si on veut comprendre ce texte, il faut avoir une idée de ce qui se faisait autour et à l'époque, lire ce qui existait en poésie, par exemple la poésie géorgienne, Edward Thomas, la poésie de la guerre aussi, Rupert Brooke, Owen... On voit alors que la rupture est là ; mais il faut avoir le sens de ce contexte historique pour s'en apercevoir. C'est pour cela qu'il faut essayer de retrouver les critiques de l'époque, la pensée de l'époque, ça permet de voir à quel point The Waste Land était moderne.

\section{NOTES}

1. McLuhan, Marshall. «Pound's Critical Prose. » The Interior Landscape: The Literary Criticism of Marshall McLuhan. New York : McGraw-Hill, 1969. (Note de l'éditrice.)

2. Lucas, F. L. "Reviews: The Waste Land». The New Statesman, 3 novembre 1923. (Note de l'éditrice.)

3. «Ezra Pound à Paris, 1965 ». YouTube. Téléversé par kevin tapalheure, 2 février 2017, www.youtube.com/watch?v=Jrq9mrPG6So (note de l'éditrice). 


\section{AUTEURS}

\section{CHLOÉ THOMAS}

Université d'Angers

BENOÎT TADIÉ

Université Rennes 2 